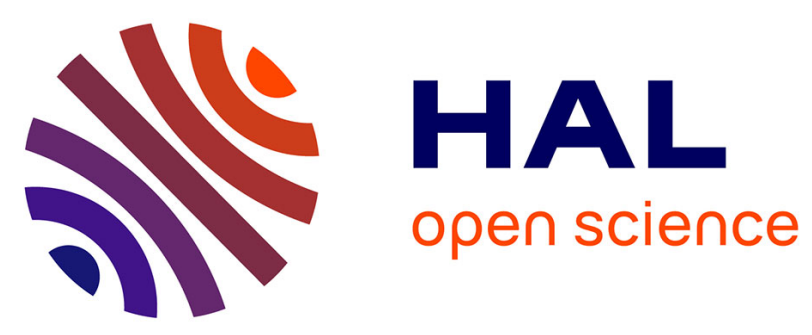

\title{
Toxoplasmic encephalitis IRIS in HIV-infected patients: a case series and review of the literature
}

\author{
Guillaume Martin-Blondel, Muriel Alvarez, Pierre Delobel, Emmanuelle \\ Uro-Coste, Lise Cuzin, Victor Cuvinciuc, Judith Fillaux, Patrice Massip, \\ Bruno Marchou
}

\section{To cite this version:}

Guillaume Martin-Blondel, Muriel Alvarez, Pierre Delobel, Emmanuelle Uro-Coste, Lise Cuzin, et al.. Toxoplasmic encephalitis IRIS in HIV-infected patients: a case series and review of the literature. Journal of Neurology, Neurosurgery and Psychiatry, 2010, 82 (6), pp.691. 10.1136/jnnp.2009.199919 . hal-00591166

\section{HAL Id: hal-00591166 https://hal.science/hal-00591166}

Submitted on 7 May 2011

HAL is a multi-disciplinary open access archive for the deposit and dissemination of scientific research documents, whether they are published or not. The documents may come from teaching and research institutions in France or abroad, or from public or private research centers.
L'archive ouverte pluridisciplinaire HAL, est destinée au dépôt et à la diffusion de documents scientifiques de niveau recherche, publiés ou non, émanant des établissements d'enseignement et de recherche français ou étrangers, des laboratoires publics ou privés. 


\section{Title page}

Toxoplasmic encephalitis IRIS in HIV-infected patients: a case series and review of the literature

Guillaume MARTIN-BLONDEL ${ }^{1,2}$, Muriel ALVAREZ ${ }^{1}$, Pierre DELOBEL ${ }^{1,2}$, Emmanuelle URO-COSTE $^{3}$, Lise CUZIN ${ }^{1}$, Victor CUVINCIUC ${ }^{4}$, Judith FILLAUX ${ }^{5}$, Patrice MASSIP ${ }^{1}$, and Bruno MARCHOU ${ }^{1}$.

${ }^{1}$ : Infectious and tropical diseases department, ${ }^{2}$ : INSERM U-563, ${ }^{4}$ : Neuroradiological department, Toulouse University Hospital, Place du Docteur Baylac TSA 40031, 31059 Toulouse cedex 9, France

${ }^{3}$ : Pathology department, ${ }^{5}$ : Parasitology department, Toulouse University Hospital, 1, avenue du Pr Jean Poulhès TSA 50032, 31059 Toulouse cedex, France

*Correspondence to: Guillaume MARTIN-BLONDEL, Infectious and tropical diseases department, Toulouse University Hospital, Place du Docteur Baylac TSA 40031, 31059 Toulouse cedex 9, France. Tel: +33 5617720 49; Fax: +33 561772138

Email: guillaumemb@gmail.com

Key words: HIV; toxoplasmic encephalitis; immune reconstitution inflammatory syndrome; chemoprophylaxis.

Word count: 1500

\section{Licence for Publication:}


I, G. Martin-Blondel, the Corresponding Author has the right to grant on behalf of all authors and does grant on behalf of all authors, an exclusive licence (or non exclusive for government employees) on a worldwide basis to the BMJ Publishing Group Ltd to permit this article (if accepted) to be published in Journal of Neurology, Neurosurgery and Psychiatry (JNNP) and any other BMJ group products and sublicences such use and exploit all subsidiary rights, as set out in our licence.

Names and e-mail addresses of all co-authors:

- Muriel ALVAREZ alvarez.m@ chu-toulouse.fr

- Pierre DELOBEL delobel.p@ chu-toulouse.fr

- Emmanuelle URO-COSTE uro-coste.m@chu-toulouse.fr

- Lise CUZIN cuzin.1@ chu-toulouse.fr

- Victor CUVINCIUC cuvinciuc.v@ chu-toulouse.fr

- Judith FILLAUX fillaux.j@ chu-toulouse.fr

- Patrice MASSIP massip.p@ chu-toulouse.fr

- Bruno MARCHOU marchou.b@chu-toulouse.fr 


\section{ABSTRACT}

Background: Toxoplasmic encephalitis associated with immune reconstitution inflammatory syndrome (TE-IRIS) is rarely described.

Methods: To identify TE-IRIS cases, we performed a retrospective study of all HIV-infected patients diagnosed with TE in our unit between January 2000 and June 2009, and a review of published cases.

Results: Three patients out of our 65 TE cases, together with six from the literature, fulfilled unmasking TE-IRIS definition. None fulfilled paradoxical TE-IRIS definition. TE occurred within a median time of 48.5 days [ $\left.\mathrm{IQ}_{25-75} 21-56\right]$ after starting antiretroviral therapy. Cases did not have distinctive clinical nor neuroimaging features from TE occurring without immune reconstitution. However: i) Cases occurred at a median CD4 lymphocytes count of $222 / \mu \mathrm{L}\left[\mathrm{IQ}_{25-75} 160-280\right]$, ii) TE occurred in five patients who were supposed to take an effective chemoprophylaxis, iii) Two patients had a brain biopsy showing an intense angiocentric inflammatory infiltrates with predominantly CD8 lymphocytes, iv) In one patient, the abnormal length of evolution under treatment might be due to the heightened immune response.

Conclusion: Although rare, unmasking TE-IRIS exists and might occur despite effective prophylaxis and an unusually high CD4 lymphocytes count. IRIS does not modify TE diagnosis and treatment but might extend its clinical course. 


\section{INTRODUCTION}

Toxoplasmic encephalitis (TE) is one of the most common life-threatening central nervous system (CNS) infections in HIV-infected patients with advanced disease. [1] TE's incidence has decreased substantially with antiretroviral therapy (ART) and broad use of cotrimoxazole prophylaxis. [2] However, TE occurring shortly after starting ART has been described. [3] Opportunistic infections occurring during the first months of ART largely reflect persistent immunodeficiency, but in some patients, immune reconstitution inflammatory syndrome (IRIS) may influence their clinical presentation and course. [4] We report three cases of TE-IRIS and a review of the literature.

\section{METHODS}

We performed a retrospective study of all HIV-infected patients diagnosed with TE in Toulouse University Hospital, France, between January 2000 and June 2009. TE diagnosis was established by the presence of suggestive clinical and neuroimaging features in a patient seropositive for Toxoplasma gondii and a favorable response to specific antibiotic regimens in the absence of other opportunistic infections. When available, characteristic histopathologic features in brain tissue confirmed the diagnosis. TE-IRIS was defined by the following criteria: i) absence of neurological complaints in a patient starting ART and presenting afterward with neurological abnormalities due to TE (unmasking TE-IRIS), or pre-existing TE worsening after ART initiation (paradoxical TE-IRIS); ii) treatment with ART resulting in a decrease in plasma HIV viral load (VL) $>1 \log _{10}$ at the onset of neurological symptoms; iii) symptoms could not be explained by another newly acquired infection, the expected course of 
TE, nor drug toxicity. We collected information on clinical features, microbiologic and virologic analyses, neuroimaging, pathology, treatment, and outcome.

\section{RESULTS}

\section{Case reports}

Three cases fulfilled the criteria for unmasking TE-IRIS among 65 TE cases diagnosed during the 9-year study period. The other 62 TE cases were all diagnosed prior to ART initiation or in the context of virological failure. Among them, 26 did not develop paradoxical TE-IRIS despite an effective ART. The other cases could not be classified due to lack of virological control and/or short follow-up. Three male patients were admitted with Pneumocystis jiroveci pneumonia $(\mathrm{PjP})$ revealing HIV-1 infection. Their CD4 lymphocyte count was respectively 9 , 25 and $23 / \mu \mathrm{L}$ and their plasma VL 5.9, 5.1 and $5.5 \log _{10}$ copies/mL. They improved on parenteral cotrimoxazole therapy, secondarily replaced by atovaquone or pentamidine aerosol at day 10,15 , or 21 due to intolerance. Primary prophylaxis against $T$. gondii was provided by pyrimethamine/atovaquone in two cases and pyrimethamine alone in the last one. Boosted protease inhibitor (PI) based ART was then started. Eighteen, 43 and 48 days later, they developed seizures with fever associated with hemiparesis for two of them. Brain MRI showed unique or multiple ring enhancing abscess (figure 1A). CSF protein level and cell count were normal, and testing for all pathogens were negative. Blood anti-Toxoplasma IgG was positive in the 3 cases. CD4 lymphocytes count were respectively 101, 191 and $222 / \mu \mathrm{L}$ and plasma VL had decreased by more than $2 \log _{10}$ copies/mL at the onset of symptoms. ART was continued and empiric pyrimethamine and clindamycin were initiated for suspected TE. For the first patient, in the absence of clinical improvement after one month of treatment, a stereotactic brain biopsy of the right occipital lobe was performed. Neuropathologic examination showed a necrotic central area with an epithelioid granuloma and a marked 
intraparenchymal lymphocytic infiltration by CD8 lymphocytes at the periphery, suggestive of a recovered immune response (Figure $1 \mathrm{C}$ and 1D). Direct examination and PCR were negative for Toxoplasma, probably because biopsy was realized after one month of clindamycine/pyrimethamine. In this case, diagnosis of $\mathrm{TE}$ was probabilistic and retrospectively reinforced by the favorable outcome with specific antibiotic regimens. After one year, clinical condition and brain MRI (Figure 1B) gradually improved for all patients, with residual episodic seizures for two of them.

\section{Characteristics of the patients}

Three patients described above as well as six from the literature fulfilled unmasking TE-IRIS definition. (5-8) Among these nine patients (table 1, online supplement), median age was 42 years [IQ $25-7536-45], 89 \%$ were male, and 78\% were ART naïve. ART (based on boosted PI in four cases, PI in four cases, and quadruple nucleoside reverse transcriptase inhibitors in one case) was started because of PjP revealing HIV infection in three cases and because of CD4 lymphocytes count $<200 / \mathrm{mm}^{3}$ in five cases. Median CD4 lymphocytes count and plasma VL at baseline were $80 / \mu \mathrm{L}$ [IQ $\mathrm{IQ}_{25-75}$ 23-130] and $5.2 \log _{10}$ copies/mL [IQ $25-75$ 5.1-5.5]. Chemoprophylaxis against $T$. gondii was pyrimethamine/atovaquone in two cases, cotrimoxazole in two cases, pyrimethamine only in one case, none in three cases, and unknown in one case. TE-IRIS was identified within a median time of 48.5 days [IQ $_{25-75} 21$ 56]. Median CD4 lymphocytes count at IRIS was $222 / \mu \mathrm{L}$ [IQ $\left.{ }_{25-75} 160-280\right]$ with a median increase of $120 / \mu \mathrm{L}$ [ $\left.\mathrm{IQ}_{25-75} 80-160\right]$ from baseline. Median plasma VL was $3.3 \log _{10}$ copies/mL [ $\left.\mathrm{IQ}_{25-75} 2.42-3.32\right]$ with a median decrease of $2.2 \log _{10}$ copies/mL [IQ $25-75$ 1.9-3.5] since ART initiation. Clinical presentation was paresis in six cases, seizure in five cases, fever in two cases, and choreiform movements in one case. CSF analysis was normal in the four cases reported. Brain MRI showed unique (two cases) or multiple (five cases) ring enhancing 
abscesses, T2 signal abnormality with unusual speckled pattern of gadolinium enhancement in one case, and was not described in one case. Another patient underwent brain biopsy showing intense angiocentric inflammatory infiltrates with predominantly CD8 lymphocytes in the presence of tachyzoïtes, testifying active toxoplasmosis. [5] Four patients received clindamycine and pyrimethamine, four cotrimoxazole, and one atovaquone. None received steroids. Seizure or residual deficit persisted in three cases.

\section{DISCUSSION}

While CNS IRIS caused by mycobacteria, cryptococci, and JC virus are increasingly being recognized, [9] TE-IRIS is by contrast rare. Although the incidence of TE decreased, TE remains a common opportunistic infection, suggesting that $T$. gondii is an infrequent cause of IRIS. Intracellular survival strategy and immune evasion tools developed by $T$. gondii might explain why unmasking TE-IRIS is uncommon. Decreased expression of immunogenic surface proteins and low-metabolism might allow bradyzoites to remain hidden from the host's immune system. [10] Interference with the MHC class I and class II antigen presentation pathway and Interferon- $\gamma$ signalling, [11] could attenuate the intensity of the cellmediated immune response during reconstitution on ART. Such as paradoxical IRIS represents an inflammatory response to a non-viable pathogen once the infection is treated and as it is unlikely that a non-viable pathogen could interfere with immune response, the reason for paradoxical TE-IRIS not being described is not understood.

Herein nine cases of TE fulfilling criteria for unmasking IRIS are presented. A critical question is whether TE occurring shortly after ART represents unmasking IRIS or persistence of immunodeficiency. [4] Cases did not have distinctive clinical nor neuroimaging features 
from TE occurring without immune reconstitution. However some features were suggestive of unmasking TE-IRIS for seven cases: i) occurrence despite an effective chemoprophylaxis and an unusually high CD4 lymphocytes count, ii) histopathologic features showing evidence of a vigorous immune response with a predominance of CD8 lymphocytes infiltration who seems to be unusual before immune reconstitution [12] and iii) the unusual protracted course under treatment in our patient, possibly due to the heightened immune response. In two cases occurring without these features, [7,8] it is difficult to state whether the presentation was related to IRIS or to persistent immunodeficiency in the absence of other clinical or neuroimaging features. In fact, both explanations are not exclusive and partly explain the course of the disease. Furthermore, some could argue that because management of unmasking TE-IRIS is not different from management of the OI, notably because steroids have never been used, making such a differentiation not crucial.

We did not identify any case fulfilling paradoxical TE-IRIS definition. A single case of paradoxical worsening of a TE following ART initiation, contemporary to a significant CD4 cell count increase has been reported. [13] However, immunohistochemical analysis of a brain biopsy revealed tachyzoites in cerebral parenchyma, which is a replicative viable form of $T$. gondii. Moreover examination of affected tissue did not reveal evidence of an immune response (such as infiltrating lymphocytes or granulomatous inflammation). This description is more suggestive of an unfavorable course of a previously diagnosed TE than of paradoxical TE-IRIS. Worsening of a treated TE after ART beginning must therefore lead first to search for differential diagnosis or inappropriate treatment. Any doubt should lead to a brain biopsy to preclude other diagnosis such as lymphoma.

In latently infected patients a subclinical replication probably persisted, despite the fact that they were supposed to take an effective chemoprophylaxis. Tachyzoïte production 
might be responsible for subsequent exacerbation of a specific cell-mediated response during immune reconstitution. This underlines the importance of prescription and adherence to an effective chemoprophylaxis against $T$. gondii when initiating ART. In patients who develop hypersensitivity to cotrimoxazole, desensitization or the recommended alternative dapsonepyrimethamine is warranted.

In conclusion, although rare, TE might occur during immune reconstitution as an unmasking IRIS, despite the prescription of an effective prophylaxis and an unusually high CD4 lymphocytes count. Furthermore, IRIS does not modify TE diagnosis and treatment, but might extend its usual clinical course. 


\section{LEGENDS}

Table 1 (online supplement): Main characteristics of unmasking TE-IRIS cases

3TC: Lamivudine; ABC: Abacavir; BPI: Boosted protease inhibitor; ddI: Didanosine; FTC: Emtricitabine; FPV: Fosamprenavir; IDV: Indinavir; LPV: Lopinavir; PI: Protease inhibitor; NRTI: Nucleoside reverse transcriptase inhibitor; M: Man; NFV: Nelfinavir; PjP: Pneumocystis jiroveci pneumonia; RTV: Ritonavir; d4T: Stavudine; TDF: Tenofovir; W: Woman; ZDV: Zidovudine.

Figure 1: MRI and histological aspects of unmasking TE-IRIS

A: Axial gadolinium-enhanced T1 MRI image at the time of TE diagnosis: right occipital ring-like enhancement.

B: Axial gadolinium-enhanced T1 MRI image at one-year follow-up: no enhancement, cortical and subcortical right occipital sequelae.

C: Cerebral biopsy of the necrotic central part of the lesion. In the left field, macrophagic resorption of necrotic tissue. In the right field, a rounded and organized epithelioid granuloma. Direct examination and PCR was negative for Toxoplasma gondii (Hematoxylin and eosin staining, original magnification $\mathrm{x} 20$ ).

D: Cerebral biopsy of the perinecrotic part of the lesion. Lymphocytic infiltration of brain tissue, particularly in the perivascular area. Marked perivascular lymphocytic CD8 infiltrate (brown nuclei). Endothelial cell hyperplasia (CD8 immunohistochemistry, original magnification $\mathrm{x} 40)$. 
Acknowledgements: many thanks to Drs. V. Soriano, G. Pfeffer, B. Ledergerber and D. Jevtovic for providing additional details on their published cases and to Dr N. Blanchard for reading the manuscript and for helpful discussion.

\section{No financial disclosure}




\section{REFERENCES}

1. Antinori A, Larussa D, Cingolani A, Lorenzini P, Bossolasco S, Finazzi MG, et al. Prevalence, associated factors, and prognostic determinants of AIDS-related toxoplasmic encephalitis in the era of advanced highly active antiretroviral therapy. Clin Infect Dis 2004; $39: 1681-91$.

2. Abgrall S, Rabaud C, Costagliola D. Incidence and risk factors for toxoplasmic encephalitis in human immunodeficiency virus-infected patients before and during the highly active antiretroviral therapy era. Clin Infect Dis 2001; 33:1747-55.

3. Michelet C, Arvieux C, Francois C, Besnier JM, Rogez JP, Breux JP, et al. Opportunistic infections occurring during highly active antiretroviral treatment. AIDS 1998; $12: 1815-22$.

4. French MA. HIV/AIDS: immune reconstitution inflammatory syndrome: a reappraisal. Clin Infect Dis 2009; 48:101-7.

5. Pfeffer G, Prout A, Hooge J, Maguire J. Biopsy-proven immune reconstitution syndrome in a patient with AIDS and cerebral toxoplasmosis. Neurology 2009; 73:321-2.

6. Tsambiras PE, Larkin JA, Houston SH. Case report. Toxoplasma encephalitis after initiation of HAART. AIDS Read 2001; 11:608-10.

7. McCombe JA, Auer RN, Maingat FG, Houston S, Gill MJ, Power C. Neurologic immune reconstitution inflammatory syndrome in HIV/AIDS: outcome and epidemiology. Neurology 2009; 72:835-41.

8. Rodriguez-Rosado R, Soriano V, Dona C, Gonzalez-Lahoz J. Opportunistic infections shortly after beginning highly active antiretroviral therapy. Antivir Ther 1998; 3:229-31. 
9. Riedel DJ, Pardo CA, McArthur J, Nath A. Therapy Insight: CNS manifestations of HIV-associated immune reconstitution inflammatory syndrome. Nat Clin Pract Neurol 2006; 2:557-65.

10. Lyons RE, McLeod R, Roberts CW. Toxoplasma gondii tachyzoite-bradyzoite interconversion. Trends Parasitol 2002; 18:198-201.

11. Blader IJ, Saeij JP. Communication between Toxoplasma gondii and its host: impact on parasite growth, development, immune evasion, and virulence. APMIS 2009; 117:458-76.

12. Farkash AE, Maccabee PJ, Sher JH, Landesman SH, Hotson G. CNS toxoplasmosis in acquired immune deficiency syndrome: a clinical-pathological-radiological review of 12 cases. J Neurol Neurosurg Psychiatry 1986; 49:744-8.

13. Tremont-Lukats IW, Garciarena P, Juarbe R, El-Abassi RN. The immune inflammatory reconstitution syndrome and central nervous system toxoplasmosis. Ann Intern Med 2009; 150:656-7. 


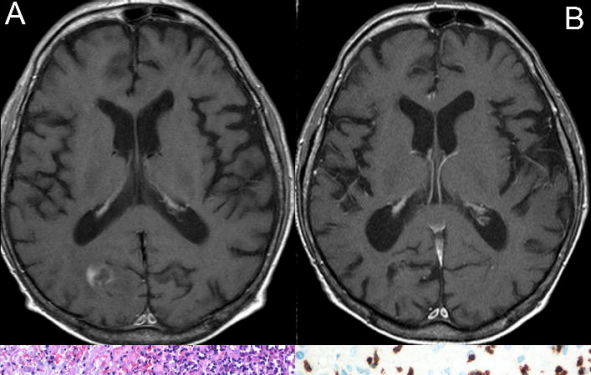

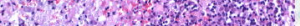

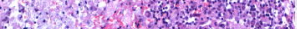
ita n. X.

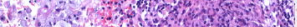

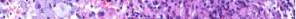

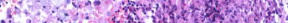
s.t.

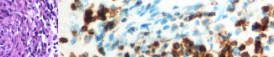
$4 \times 10$ (3)

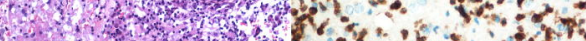

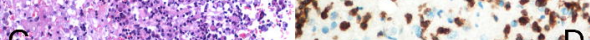
Q 